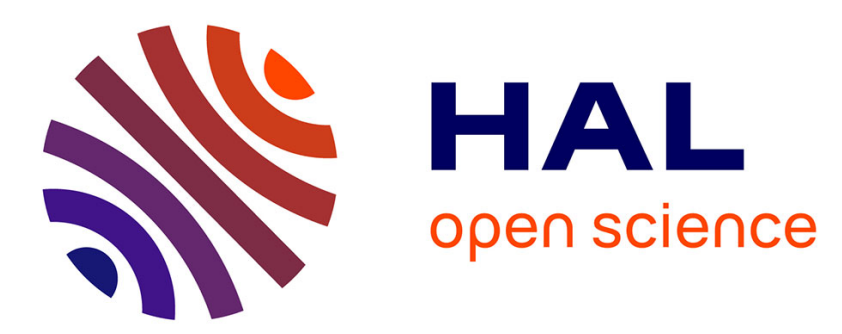

\title{
Disorder-induced losses in planar photonic crystals
}

Rolando Ferrini, Daniel Leuenberger, Romuald Houdré, Henri Benisty, Martin

Kamp, Alfred Forchel

\section{To cite this version:}

Rolando Ferrini, Daniel Leuenberger, Romuald Houdré, Henri Benisty, Martin Kamp, et al.. Disorder-induced losses in planar photonic crystals. Optics Letters, 2006, 31 (10), pp.1426-1428. 10.1364/OL.31.001426 . hal-00878202

\section{HAL Id: hal-00878202 \\ https://hal-iogs.archives-ouvertes.fr/hal-00878202}

Submitted on 29 Oct 2013

HAL is a multi-disciplinary open access archive for the deposit and dissemination of scientific research documents, whether they are published or not. The documents may come from teaching and research institutions in France or abroad, or from public or private research centers.
L'archive ouverte pluridisciplinaire HAL, est destinée au dépôt et à la diffusion de documents scientifiques de niveau recherche, publiés ou non, émanant des établissements d'enseignement et de recherche français ou étrangers, des laboratoires publics ou privés. 


\title{
Disorder-induced losses in planar photonic crystals
}

\author{
R. Ferrini, ${ }^{*}$ D. Leuenberger, ${ }^{\dagger}$ and R. Houdré \\ Institut de Photonique et d'Electronique Quantique, Ecole Polytechnique Fédérale de Lausanne, \\ CH-1015 Lausanne, Switzerland \\ H. Benisty \\ Laboratoire Charles Fabry de l'Institut d'Optique, F-91403 Orsay cedex, France \\ M. Kamp and A. Forchel \\ Technische Physik, Universität Würzburg, Am Hubland, D-97074, Würzburg, Germany
}

\begin{abstract}
Received December 1, 2005; revised January 30, 2006; accepted February 8, 2006; posted February 21, 2006 (Doc. ID 66388)
The phenomenological approach introduced by Benisty et al. [Appl. Phys. Lett. 76, 532 (2000)] to model out-of-plane radiation losses in planar photonic crystals with a low vertical refractive index contrast is extended to the case of in-plane disorder. The model is experimentally validated by means of optical measurements on GaAs-based structures. For the present fabrication techniques the disorder-induced contribution is found to be negligible compared with the other loss mechanisms. (C) 2006 Optical Society of America OCIS codes: $130.0130,130.3130,230.3120,250.5300,290.5880$.
\end{abstract}

Disorder-induced radiation losses can strongly limit the performances of planar photonic crystals $(\mathrm{PhCs})$ in actual optoelectronic devices. ${ }^{1-4}$ With the progress in lithography and etching, the effects of fabrication tolerances and imperfections are investigated to explore the limits of $\mathrm{PhC}$ structures. Disorder in the hole geometry can lead to a reduction of the bandgap size $^{1}$ and to an increase of radiation losses. ${ }^{3,4}$ Several studies both on in-plane (i.e., random displacement from the ideal periodic lattice ${ }^{3,4}$ ) and on vertical (i.e., sidewall roughness ${ }^{2}$ ) disorder have been reported. In this Letter we show how disorder-induced losses in planar PhCs can be described by adapting the phenomenological model of Refs. 5 and 6. The model is experimentally validated and the relative contributions of intrinsic, depth- and disorder-induced losses are discussed.

In the seminal paper by Benisty et $a .^{5}$ a twodimensional (2D) phenomenological approach was introduced to account for radiation losses in lowrefractive-index-contrast planar PhCs. The out-ofplane scattering at the air holes is translated into an effective dissipation, i.e., into an imaginary dielectric constant $\epsilon^{\prime \prime}$ in the air holes. ${ }^{5}$ To first order, $\epsilon^{\prime \prime}$ is the sum of three contributions:

$$
\epsilon^{\prime \prime}=\epsilon_{\mathrm{int}}^{\prime \prime}+\epsilon_{\mathrm{fed}}^{\prime \prime}+\epsilon_{\mathrm{dis}}^{\prime \prime},
$$

where $\epsilon_{\text {int }}^{\prime \prime}$ accounts for intrinsic losses, ${ }^{5} \epsilon_{\text {fed }}^{\prime \prime}$ contains contributions from the finite etch depth or, more generally, from the hole sidewall shape, ${ }^{6}$ while $\epsilon_{\text {dis }}^{\prime \prime}$, accounting for disorder, is the parameter of interest here.

Concerning intrinsic radiation losses, for most practical cases, the general scaling law of Ref. 5 holds:

$$
\epsilon_{\text {int }}^{\prime \prime} \propto \frac{n_{2} \Delta \epsilon^{2}}{\lambda_{0}^{3}} w S \Gamma_{1} \eta,
$$

where $\lambda_{0}$ is the vacuum wavelength, $w$ is the core thickness [see Fig. 1(a)], $S$ is the area of the hole cross section [see Fig. 1(c)], $\Gamma_{1}$ is the confinement factor of the guided mode, ${ }^{5}$ and $\eta \cong 1-\cos \theta$ [with $\theta$ $\left.=\arcsin \left(n_{1} / n_{2}\right)\right]$ is the extraction coefficient from the core into the claddings.

An analytical expression can be derived for the effect of the finite etch depth, ${ }^{6}$ i.e.,

$$
\epsilon_{\mathrm{fed}}^{\prime \prime}=\frac{4}{3} \pi^{2} \frac{n_{1}\left(1-n_{1}^{2}\right)^{2}}{\lambda_{0}^{3}} 2 L_{d} S \Gamma,
$$

where $L_{d}$ is the decay length of the guided mode into the bottom cladding and $\Gamma$ is the partial confinement factor, i.e., the overlap integral between $\zeta^{2}(z)$ and the underetched air hole region.

The same phenomenological approach is used here to model disorder-induced radiation losses. Positional and geometrical in-plane disorder are considered [see Figs. 1(b) and 1(c)]. In this case out-of-plane losses
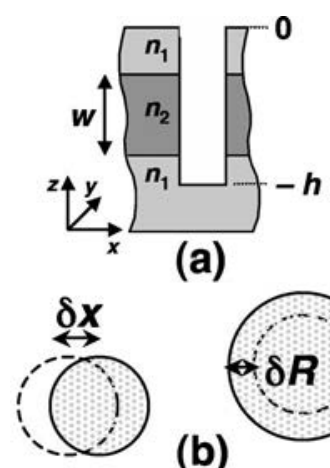

(a)
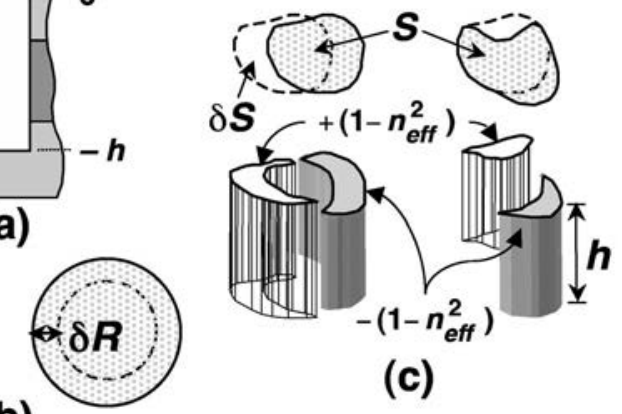

(c)

(b)

Fig. 1. Schematic of (a) a planar $\mathrm{PhC}$ etched through a vertical waveguide with a refractive index profile $n_{1} / n_{2} / n_{1}$ ( $w$, core thickness; $h$, hole depth); (b) and (c) In-plane positional (left) and geometrical (right) disorder effects ( $x$ and $R$, hole position and radius; $S$, area of the hole cross section). The perturbation with respect to the periodic lattice is represented by plugs of dielectric constant $\pm\left(1-n_{\text {eff }}^{2}\right)$ that extend down to the hole bottom $h$ with a cross-section area $\delta S$. 
can be evaluated considering the actual $\mathrm{PhC}$ as the sum of an ideal periodic array of evenly shaped holes plus a dielectric perturbation $\delta \epsilon= \pm\left(1-n_{\text {eff }}^{2}\right)$ that extends down to the hole bottom $h$ with a cross-section area of $\delta|S|=4 R \delta x$ and $2 \pi \delta R$ for positional and size (uniform expansion) disorder, respectively [see Fig. 1(b)]. The same approach as in Refs. 5 and 6 is adopted (see Appendix A), yielding

$$
\epsilon_{\mathrm{dis}}^{\prime \prime}=\frac{4}{3} \pi^{2} \frac{n_{\mathrm{eff}}\left(1-n_{\mathrm{eff}}^{2}\right)^{2}}{\lambda_{0}^{3}} w_{\mathrm{eff}} S\left(\frac{\delta|S|}{S}\right)^{2}(1-\Gamma) \eta^{\prime} .
$$

Moreover, for most etching techniques, $h$ almost overlaps with the entire $\zeta(z)$, so that $\Gamma \ll 1, \epsilon_{\text {fed }}^{\prime \prime}$ becomes negligible, and $1-\Gamma \approx 1$. ${ }^{7}$

To validate the present model, planar $\mathrm{PhCs}$ consisting of a triangular lattice of air holes were etched through a nominally undoped GaAs-based vertical waveguide grown by molecular beam epitaxy $(w$ $=240 \mathrm{~nm}) .{ }^{8}$ At the wavelengths of interest $\left[\lambda_{0}\right.$ $\approx 1050 \mathrm{~nm}: n_{1}=3.39$ (upper cladding) and 3.06 (bottom cladding), $n_{2}=3.48$ ] the effective refractive index of the fundamental transverse-electric (TE) guided mode is $n_{\text {eff }}=3.36$. Moreover, $w_{\text {eff }} \approx 650 \mathrm{~nm}, L_{d}$ $=100 \mathrm{~nm}$, and $\Gamma_{1}=0.55,{ }^{5}$ while $\eta \approx 0.50$ and $\eta^{\prime} \approx 0.04$. The $\mathrm{PhC}$ structures were fabricated by electron beam lithography and reactive ion etching. ${ }^{8}$ Simple eightrows-thick $\mathrm{PhC}$ slabs were fabricated with a lattice period $a=190-370 \mathrm{~nm}(\Delta a=20 \mathrm{~nm})$ and $h \approx 1 \mu \mathrm{m}$ (i.e., $\left.\Gamma \approx 10^{-4}\right)^{6}$ A strong in-plane disorder was deliberately introduced without any intentional disorder in the vertical direction by a nonoptimized transfer of the $\mathrm{PhC}$ pattern from the e-beam-written resist to the $\mathrm{SiO}_{2}$ mask $^{8}$ [see the top and edge scanning electron microscopy (SEM) micrographs in the inset of Fig. 2(a)]. By using an internal light source technique $^{9}$ the absolute transmission through the $\mathrm{PhC}$ structures was measured along the $\Gamma \mathrm{M}$ direction as a function of the normalized frequency $u=a / \lambda_{0}$. The $\Gamma \mathrm{M}$ transmission spectrum is shown in Fig. 2(a) (solid curves) for the TE polarization. It was fitted by a 2D finite-difference time domain (2D-FDTD) model assuming $n_{\text {eff }}$ as the matrix index. ${ }^{9}$ Out-of-plane losses were included in the 2D model by adding $\epsilon^{\prime \prime}$ in the holes. ${ }^{6}$ The fit parameters were the air filling factor $f$ and $\epsilon^{\prime \prime}$, and the calculated spectra are shown in Fig. 2(a) (gray dotted curves). The values $f$ $=0.32 \pm 0.02$ (i.e., $S \approx 2 \times 10^{4} \mathrm{~nm}^{2}$ for $a=270 \mathrm{~nm}$ ) and $\epsilon^{\prime \prime}[$ exp. $]=0.11 \pm 0.01$ were found. The latter value agrees well with the theoretical value $\epsilon^{\prime \prime}[$ th.] $=0.115 \pm 0.005$ obtained from Eqs. (1) - (4) (see Table 1). The amount of disorder $\delta|S| / S=0.2$ was found by the 2D Fourier analysis of the top SEM micrograph in Fig. 2(a), which allows the extraction of its both periodic and disordered components [Figs. 2(b) and 2(c), respectively]. Once the micrograph has been digitalized, the inverse transform of the periodic part of its Fourier transform yields the periodic $\mathrm{PhC}$ pattern, while the disordered component is obtained by subtracting the latter pattern from the total image. The very good agreement between the theory and the

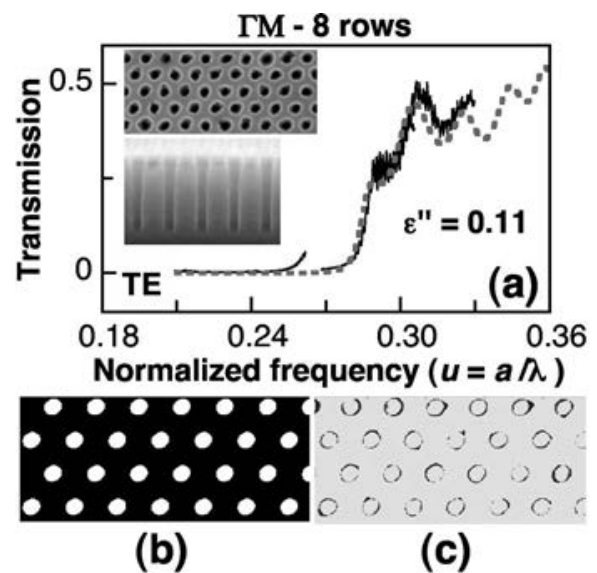

Fig. 2. (a) Measured (solid curves) and calculated (gray dotted curves) $\Gamma M$ transmission spectra through eight-rowthick disordered $\mathrm{PhC}$ slabs for TE polarization: the value of the loss parameter $\epsilon^{\prime \prime}$ is reported; the inset shows the top and edge SEM micrographs. (b) Periodic and (c) and disordered components of the top SEM image.

Table 1. Calculated and Measured Radiation Losses

\begin{tabular}{ccccc}
\hline \multicolumn{4}{c}{ Theory } & \\
\cline { 1 - 4 }$\epsilon_{\text {inf }}^{\prime \prime a}$ & $\epsilon_{\text {fed }}^{\prime \prime}$ & $\epsilon_{\text {dis }}^{\prime \prime}$ & $\epsilon^{\prime \prime}[$ th.] & $\begin{array}{c}\text { Experiment } \\
\epsilon^{\prime \prime} \text { [exp.] }\end{array}$ \\
\hline $0.035 \pm 0.005$ & 0.001 & 0.08 & $0.115 \pm 0.005$ & $0.11 \pm 0.01$ \\
\hline
\end{tabular}

${ }^{a}$ From Eq. (2) and Ref. 5.

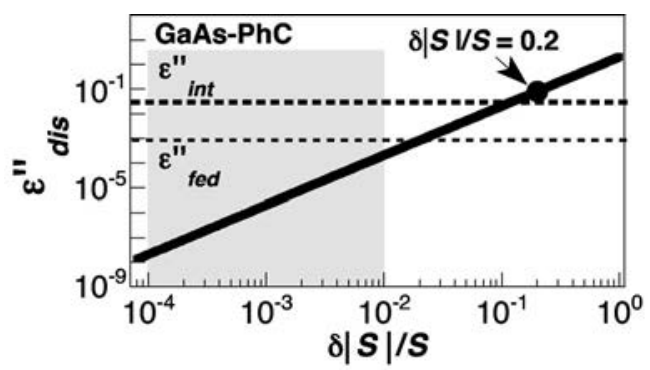

Fig. 3. Disorder-induced losses $\left(\epsilon_{\text {dis }}^{\prime \prime}\right)$ as a function of the disorder parameter $\delta|S| / S$ for GaAs-based PhCs. Circle: the disordered $\mathrm{PhC}$ shown in Fig. 2. Dotted lines and gray region: $\epsilon_{\mathrm{int}}^{\prime \prime} / \epsilon_{\mathrm{fed}}^{\prime \prime}$ and $\delta|S| / S$ values, respectively, for the present fabrication techniques of GaAs-based PhCs.

experiments validates the use of this model for the study of disorder-induced losses in planar PhCs.

In Fig. 3, Eq. (4) is plotted as a function of the disorder parameter $\delta|S| / S$ for the investigated GaAsbased PhCs, thus providing a figure of merit for the minimization of the disorder contribution with respect to the other loss sources. The filled circle in Fig. 3 corresponds to the disordered $\mathrm{PhC}$ of Fig. 2 (i.e., $\delta|S| / S=0.2$ ). The dotted lines in Fig. 3 indicate the values of $\epsilon_{\text {int }}^{\prime \prime}$ and $\epsilon_{\text {fed }}^{\prime \prime}$ calculated from Eqs. (2) and (3), respectively, for the best fabrication technologies presently available for GaAs-based $\mathrm{PhCs}$ (see also Table 1). These technologies also guarantee that $\delta R / R \approx 1 \%$ and $\delta x / x \leqslant 0.5 \%$. ${ }^{10}$ Thus for typical GaAsbased structures $(a=200-400 \mathrm{~nm}$ and $f \approx 0.3$, i.e., $R$ $\approx 60-120 \mathrm{~nm}), \delta|S| / S$ ranges from $10^{-4}$ to $10^{-2}$ for the 
geometrical and positional disorder, respectively (the gray region in Fig. 3). ${ }^{10}$ This results in disorderinduced losses that are negligible with respect to both intrinsic $\left(\epsilon_{\mathrm{dis}}^{\prime \prime} \ll \epsilon_{\text {int }}^{\prime \prime}\right)$ and depth-induced $\left(\epsilon_{\mathrm{dis}}^{\prime \prime}\right.$ $\left.<\epsilon_{\text {fed }}^{\prime \prime}\right)$ losses.

In conclusion, a quantitative analysis of disorderinduced losses in low-refractive-index-contrast planar $\mathrm{PhCs}$ has been presented. For the present fabrication techniques of GaAs-based $\mathrm{PhCs}$ the disorderinduced contribution is found to be negligible with respect to the other loss mechanisms. Concerning high-refractive-index-contrast planar $\mathrm{PhCs}$, we note that, to our knowledge, while there are several papers dealing with propagation losses in membranelike line-defect waveguides with disorder, ${ }^{11}$ little work has been done on simple PhC slabs on membranes. We believe that in such systems our phenomenological approach may be applicable and relevant for broad families of $2 \mathrm{D} \mathrm{PhC}$ bands for which the assumptions on light extraction ${ }^{5}$ are still valid and no singularity is present. These include low groupvelocity modes above the light line (some of them having indeed low intrinsic losses near the $\Gamma$ point due to cancellations that vanish in the presence of disorder). Nevertheless, based on the work on waveguides, ${ }^{12,13}$ the case of slow group-velocity modes below the light line should be treated separately.

\section{Appendix A}

Disorder-induced out-of-plane losses are generated by the radiation of the polarization $P(x, y, z)= \pm \epsilon_{0}(1$ $-n_{\text {eff }}^{2} E(x, y, z) \mathrm{d} x \mathrm{~d} y \mathrm{~d} z$ [where $E(x, y, z)=\psi(x, y) \zeta(z)$ is the separable electric field] ${ }^{5,6}$ Following Ref. 6 , the power radiated in the homogeneous medium of refractive index $n_{\text {eff }}$ and then lost into the claddings can be written as ${ }^{5,6}$

$$
\begin{aligned}
P_{\mathrm{rad}}= & \frac{4}{3} \pi^{3} \frac{c}{\lambda_{0}^{4}} \epsilon_{0} n_{\mathrm{eff}}\left(1-n_{\mathrm{eff}}^{2}\right)^{2} \\
& \times S\left(\frac{\delta|S|}{S}\right)^{2} \iint_{S} \psi^{2}(x, y) \mathrm{d} x \mathrm{~d} y\left[\int_{-h}^{0} \zeta(z) \mathrm{d} z\right]^{2} \eta^{\prime},
\end{aligned}
$$

where $c$ and $\epsilon_{0}$ are the vacuum light speed and dielectric constant, respectively, and $\eta^{\prime} \cong 1-\cos \theta^{\prime}$ [with $\left.\theta^{\prime}=\arcsin \left(1 / n_{\text {eff }}\right)\right]$ is the extraction coefficient from the waveguide slab to the air. The approximation

$$
\left[\iint_{\delta S} \psi(x, y) \mathrm{d} x \mathrm{~d} y\right]^{2} \cong S(\delta|S| / S)^{2} \iint_{S} \psi^{2}(x, y) \mathrm{d} x \mathrm{~d} y
$$

is made. ${ }^{6}$ The disorder-induced losses per hole can be represented as the dissipated power ${ }^{5,6}$

$$
P_{\text {diss }}=\frac{1}{2} \epsilon_{0} \frac{2 \pi c}{\lambda_{0}} \epsilon_{\mathrm{dis}}^{\prime \prime} \iint_{S} \psi^{2}(x, y) \mathrm{d} x \mathrm{~d} y \int_{-\infty}^{+\infty} \zeta^{2}(z) \mathrm{d} z .
$$

Finally, for $h \gg w+L_{\mathrm{d}}$, the effective width of the guided mode can be defined as

$$
\begin{aligned}
w_{\text {eff }} & \cong\left[\int_{h}^{+\infty} \zeta(z) \mathrm{d} z\right]^{2} / \int_{h}^{+\infty} \zeta^{2}(z) \mathrm{d} z \\
& \cong\left[\int_{-\infty}^{+\infty} \zeta(z) \mathrm{d} z\right]^{2} / \int_{-\infty}^{+\infty} \zeta^{2}(z) \mathrm{d} z .
\end{aligned}
$$

Thus equating Eqs. (A1) and (A2) yields Eq. (4).

The authors thank William Whelan-Curtin, Saint Andrews University, for discussions and data on fabrication tolerances. The work was done in the framework of IST project PCIC (IST-1999-11239) and the European Network of Excellence on Photonic Integrated Components and Circuits (ePIXnet-contract 004525). R. Ferrini's e-mail address is rolando.ferrini@epfl.ch.

*Present address, Laboratoire d'Optoélectronique des Matériaux Moléculaires, École Polytechnique Fédérale de Lausanne, CH-1015 Lausanne, Switzerland.

${ }^{\dagger}$ Present address, Department of Electrical Engineering and Computer Sciences, UC Berkeley, Berkeley, California 94720-1770.

\section{References}

1. E. Lidorikis, M. M. Sigalas, E. N. Economou, and C. M. Soukoulis, Phys. Rev. B 61, 13458 (2000).

2. W. Bogaerts, P. Bienstman, and R. Baets, Opt. Lett. 28, 689 (2003).

3. M. Skorobogatiy, G. Bégin, and A. Talneau, Opt. Express 13, 2487 (2005), and references therein.

4. J. M. Rico-García, J. M. López-Alonso, and J. Alda, Opt. Express 13, 3082 (2005), and references therein.

5. H. Benisty, D. Labilloy, C. Weisbuch, C. J. M. Smith, T. F. Krauss, C. Jouanin, and D. Cassagne, Appl. Phys. Lett. 76, 532 (2000).

6. R. Ferrini, R. Houdré, H. Benisty, M. Qiu, and J. Moosbürger, J. Opt. Soc. Am. B 20, 469 (2003).

7. R. Ferrini, A. Berrier, L. A. Dunbar, R. Houdré, M. Mulot, S. Anand, S. de Rossi, and A. Talneau, Appl. Phys. Lett. 85, 3998 (2004).

8. J. Moosbürger, M. Kamp, A. Forchel, R. Ferrini, D. Leuenberger, R. Houdré, S. Anand, and J. Bergreen, Nanotechnology 13, 341 (2002).

9. R. Ferrini, D. Leuenberger, M. Mulot, M. Qiu, J. Moosbürger, M. Kamp, A. Forchel, S. Anand, and R. Houdré, Quantum Electron. 38, 786 (2002).

10. W. Whelan-Curtin, X. Yuan, and T. F. Krauss, "Effects of disorder in photonic crystals," presented at Proceedings of the PECS-VI Conference, Crete, 2005.

11. D. Gerace and L. C. Andreani, Photonics Nanostruct. Fundam. Appl. 3, 120 (2005).

12. S. Hughes, L. Ramunno, J. F. Young, and J. E. Sipe, Phys. Rev. Lett. 94, 033903 (2005).

13. E. Kuramochi, M. Notomi, S. Hughes, A. Shinya, T. Watanabe, and L. Ramunno, Phys. Rev. B 72, 161318 (2005). 